\title{
Influence of Surface Recombination on Charge-Carrier Kinetics in Organic Bulk Heterojunction Solar Cells with Nickel Oxide Interlayers
}

\author{
Scot Wheeler, ${ }^{1}$ Florent Deledalle, ${ }^{1}$ Nurlan Tokmoldin, ${ }^{1}$ Thomas Kirchartz, ${ }^{2,3, *}$ \\ Jenny Nelson, ${ }^{4}$ and James R. Durrant ${ }^{1, \dagger}$ \\ ${ }^{1}$ Department of Chemistry and Centre for Plastic Electronics, Imperial College London, \\ South Kensington Campus, SW7 2AZ London, United Kingdom \\ ${ }^{2}$ IEK5-Photovoltaics, Forschungszentrum Jülich, 52425 Jülich, Germany \\ ${ }^{3}$ Faculty of Engineering and CENIDE, University of Duisburg-Essen, \\ Carl-Benz-Strasse 199, 47057 Duisburg, Germany \\ ${ }^{4}$ Department of Physics and Centre for Plastic Electronics, Imperial College London, \\ South Kensington Campus, SW7 2AZ London, United Kingdom
}

(Received 21 May 2015; revised manuscript received 23 July 2015; published 28 August 2015)

\begin{abstract}
The choice of electrode for organic photovoltaics is known to be of importance to both device stability and performance, especially regarding the open-circuit voltage $\left(V_{\mathrm{OC}}\right)$. Here we show that the work function of a nickel oxide anode, varied using an $\mathrm{O}_{2}$ plasma treatment, has a considerable influence on the opencircuit voltage $V_{\mathrm{OC}}$ of an organic solar cell. We probe recombination in the devices using transient photovoltage and charge extraction to determine the lifetime as a function of charge-carrier concentration and compare the experimental results with numerical drift-diffusion simulations. This combination of experiment and simulations allows us to conclude that the variations in $V_{\mathrm{OC}}$ are due to a change in surface recombination, localized at the $\mathrm{NiO}$ anode, although only a small change in carrier lifetime is observed.
\end{abstract}

DOI: 10.1103/PhysRevApplied.4.024020

\section{INTRODUCTION}

Organic photovoltaics (OPV) have the potential to provide a low-cost, lightweight, and flexible alternative to solar cells based on inorganic technologies, especially for the fabrication of large area devices [1,2]. The performance and stability of OPV has steadily improved in recent years; however, at approximately $10 \%$ it is still inferior to that of inorganic technologies [3-6]. The development of electrode materials to improve performance, processing, and stability has received extensive interest recently $[7,8]$; however, the potential impact of recombination at the active-layer-electrode interfaces is yet to be fully understood. In this paper, we employ nickel oxide as an anode interlayer in OPV devices and address how changes in cell open-circuit voltage $V_{\mathrm{OC}}$, caused by modulation of the $\mathrm{NiO}$ work function, can be understood in terms of changes in charge-carrier density distributions and lifetimes in the active layer of the device.

A key parameter defining photovoltaic performance is the open-circuit voltage $V_{\mathrm{OC}}$. While the energetics of the active layer have been shown on numerous occasions to influence $V_{\mathrm{OC}}[9,10]$, this is not the only defining parameter $[11,12]$. The $V_{\mathrm{OC}}$ is often related to losses due to nongeminate recombination of separated charge carriers at

\footnotetext{
*Corresponding author. t.kirchartz@fz-juelich.de

${ }^{\dagger}$ Corresponding author. j.durrant@imperial.ac.uk
}

the polymer fullerene interface, as defined by the activelayer density of states and carrier lifetimes [13,14]. However, the $V_{\mathrm{OC}}$ of organic solar cells can also be reduced by recombination at the "wrong" electrodes, for instance, recombination of holes in the semiconductor with electrons at the cathode [15-17]. This surfacerecombination mechanism becomes more important when the electrode is nonselective. Improved control of voltage losses at the donor-acceptor heterojunction has resulted in a growing appreciation of the importance of surface recombination and the need to minimize such losses $[17,18]$.

Our study uses experimental and modeling techniques to investigate how electrodes impact the recombination kinetics at open circuit. An ideal electrode alignment is one in which the work function coincides with the HOMO or LUMO of the active layer, leading to a minimal injection barrier for majority carriers; this minimizes the equilibrium concentration of minority carriers at that electrode, thus reducing losses associated with recombination at this interface $[19,20]$. Most previous studies of $V_{\mathrm{OC}}$ focus upon the impact of nongeminate recombination at the internal donor-acceptor interface [13,21-26]. At present, it is unclear whether changes in $V_{\mathrm{OC}}$ due to surface recombination can also be understood through changes in bulk charge-carrier density and lifetime or whether gradients in the quasi-Fermi-level near the electrode interface are also important. While there are some experimental $[16,18,27,28]$ and theoretical studies into surface recombination [19,29-31], rationalizing surface recombination by combining experiment and steady-state 
modeling is more limited. In this work, we combine charge extraction (CE) and transient photovoltage (TPV) techniques, widely employed to study the impact of bulk nongeminate recombination losses at $V_{\mathrm{OC}}[12,13,22]$, with 1D drift-diffusion modeling to investigate the effects of surface recombination on the open-circuit voltage [27,31]. As a model system to investigate the influence of surface recombination, we employ $\mathrm{NiO}$ as an anodic interlayer. Metal oxide hole transporting interlayers such as $\mathrm{NiO}$ and $\mathrm{MoO}_{3}$ are popular alternatives to PEDOT:PSS, which due to its hygroscopic and acidic nature can lead to degraded electrical properties [32-34]. By utilizing the effect of an oxygen plasma treatment on $\mathrm{NiO}$ interlayers in PCDTBT: $\mathrm{PC}_{71} \mathrm{BM}$ devices, the energy offset between the interlayer and the active layer can be varied in a controlled manner [8].

\section{MODELING BACKGROUND}

At $V_{\mathrm{OC}}$, there is no net external current; therefore, a balance between charge generation and charge recombination averaged over the device exists. Nongeminate recombination dominates at open circuit due to high excess carrier densities; the recombination flux $R$ typically has an observed dependence on the excess charge-carrier density $n$ of the order of greater than 2, which has been assigned to the filling of a tail of trap states within the band gap of the active layer [35-37]. While this recombination is normally thought to occur at the internal donor-acceptor interface, charges can also recombine at the contacts, which can have a large influence on the achievable open-circuit voltage of a device. CE is commonly used to provide an in situ measurement of the variation in quasi-Fermi-level splitting with charge density, i.e., of the average of the electron and hole density of states (DOS). TPV provides a measurement of the average lifetime of carriers which are able to contribute to the voltage of the device. The recombination kinetics can be investigated by combining the two techniques to obtain the density dependence of the recombination lifetime. The measured lifetime includes both charges that recombine at the donor-acceptor interface and at the electrode, which makes it more difficult to separate the two recombination pathways. To complement the experimental data, steady-state 1D drift-diffusion simulations based on the software advanced semiconductor analysis (ASA) are used to simulate the CE and TPV measurements [19,36,38], as described in the Materials and Methods section. The plasmatreated device is modeled first, and then only the anode work function is varied to simulate the untreated device.

The current density lost through surface recombination, $J_{S}$, is usually defined in terms of the excess minority carrier concentration $\Delta n_{\min }$ (electrons at the anode) at the electrode-active-layer interface and the surfacerecombination velocity $S_{\min }$ [19]:

$$
J_{S}=q S_{\min } \Delta n_{\min },
$$

where $q$ is the elementary charge. We expect all minority carriers on reaching the wrong electrode (electrons at the anode) to recombine with a majority carrier; therefore, $S_{\text {min }}$ is expected to be large and remain constant with respect to the minority carrier density. However, it is possible that $S_{\min }$ could be $\Delta n_{\min }$ dependent, in which case the surfacerecombination current would scale nonlinearly with $\Delta n_{\min }$; such nonlinear behavior is beyond the scope of the study reported herein [19]. The effect of varying the surfacerecombination velocity for both majority and minority carriers has been modeled previously [30,31]. We note that majority carrier "surface recombination" corresponds to charge extraction to yield the desired photocurrent; as such, $S_{\text {maj }}$ needs to be high. The physical meaning of a small majority carrier surface-recombination velocity is an extraction barrier that would eventually lead to S-shaped light $J-V$ curves. Experimentally, such $\mathrm{S}$-shaped curves are not observed for the devices studied herein; however, S-shaped $J-V$ curves could be observed in simulations when $S_{\min }$ is reduced and are discussed further in Supplemental Material [39]. The excess minority carrier concentration at the cathode or anode at open circuit depends on the ability of carriers to diffuse against the built-in field, which, depending on the light intensity, is small at open circuit. Therefore, cell thickness, carrier mobility, and the built-in voltage are important factors in determining the influence of minority carrier surface recombination.

\section{RESULTS AND DISCUSSION}

\section{A. Device performance}

An oxygen plasma treatment is used to vary experimentally the work function of a nickel oxide hole transport layer in a conventional bulk heterojunction device with structure ITO/NiO/PCDTBT: $\mathrm{PC}_{71} \mathrm{BM}(1: 2) / \mathrm{Ca} / \mathrm{Al}$, as described in the experimental section and demonstrated elsewhere $[28,40]$. The work function of a $\mathrm{NiO}$ film after a 7 -min oxygen plasma treatment increased from 4.85 to $5.30 \mathrm{eV}$, a change of $450 \mathrm{meV}$, measured by a Kelvin probe [41]. This is in good agreement with previous measurements of $\mathrm{NiO}$ films by Kelvin probe and UPS techniques [8]. Plasmatreated $\mathrm{NiO}$ is therefore an attractive system for studies of surface recombination due to the large shift in work function in the range of common semiconducting polymer HOMO levels such as PCDTBT (approximately $5.3 \mathrm{eV}$ ) and P3HT (approximately $4.9 \mathrm{eV}$ ).

As observed elsewhere [8], this change in work function leads primarily to a change in the open-circuit voltage $\left(V_{\mathrm{OC}}\right)$ with only small changes in fill factor (FF) and short-circuit current density $\left(J_{\mathrm{SC}}\right)$; a summary of device performance, including power conversion efficiency (PCE), can be found in Table I. The shift in $V_{\mathrm{OC}}$ we observe is large, up to $458 \mathrm{mV}$. The $V_{\mathrm{OC}}$ of the untreated $\mathrm{NiO}$ is much lower than typically achieved for PEDOT:PSS/PCDTBT: $\mathrm{PC}_{71} \mathrm{BM}$, while the treated $\mathrm{NiO}$ has a comparable $V_{\mathrm{OC}}$. The size of the observed effect demonstrates the importance of understanding how electrodes impact on device performance and 


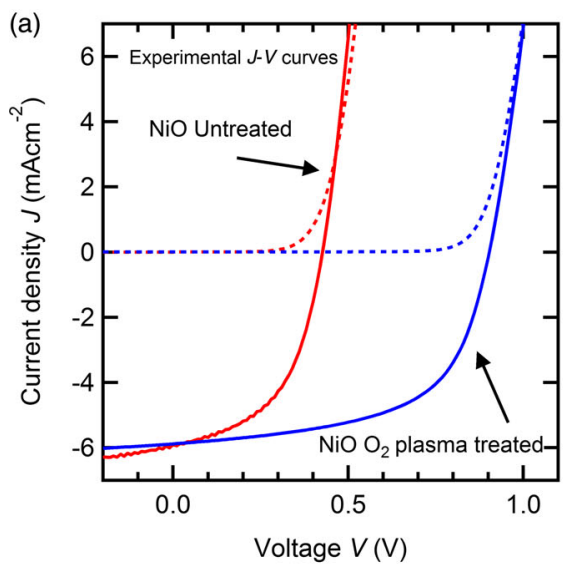

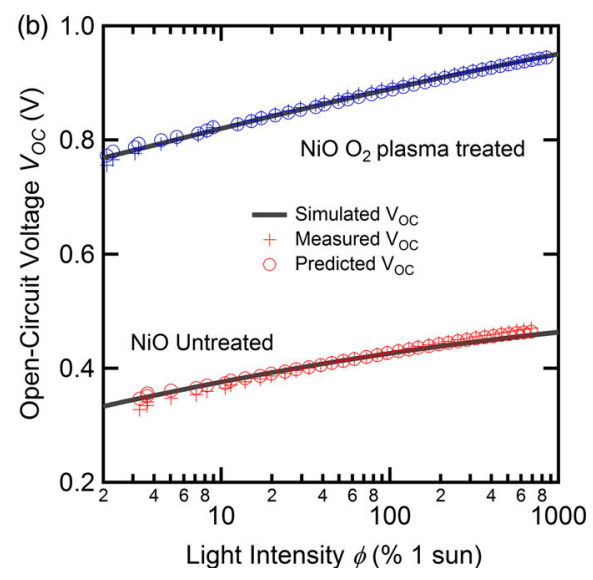

FIG. 1. (a) Dark (dashed lines) and illuminated under AM1.5 (solid lines) $J-V$ curves for devices made on untreated and $\mathrm{O}_{2}$ plasma-treated NiO. (b) Opencircuit voltage versus illumination for both devices (crosses) as well as predictions based on experimental measurements of the density dependence of charge-carrier lifetime $\tau_{n}$ (open symbols) and simulations based on 1D drift-diffusion modeling (solid lines). measurements; such a large change could easily mask other changes in active-layer design which might be of interest.

As shown in Fig. 1(b), $V_{\mathrm{OC}}$ as a function of light intensity shows approximately logarithmic behavior at low light intensity as one would expect; however, at high light intensity, the $V_{\mathrm{OC}}$ in both devices appears to start to saturate [42]. This is expected as, with increasing light intensity, the quasi-Fermi-level splitting approaches the built-in voltage $[16,25]$. One might expect the untreated device to approach this limit at a lower voltage compared to the $\mathrm{O}_{2}$ plasma-treated device. One can probe this limit in more detail using high light intensity measurements as demonstrated by Law et al. [43].

\section{B. Device simulation}

1D drift-diffusion simulations are carried out to elucidate whether the origin of the shift in open-circuit voltage with $\mathrm{O}_{2}$ plasma treatment could be explained solely in terms of a change in the electrode work function. A reasonable set of parameters is obtained from the full experimental data of the treated device following the procedure detailed in the materials and methods section and can be seen in Table II. These compare well with previously published parameters describing the PCDTBT: $\mathrm{PC}_{71} \mathrm{BM}$ system [16]. Following this, only the work function of the anode is allowed to vary when simulating the untreated device. The impact of this shift in work function upon the device simulations is found to be largely independent of the specific set of other parameters used to describe the device.

Figures 2(a) and 2(b) show band diagrams at open circuit and 1-sun illumination obtained from the model using the

TABLE I. Device performance summary.

\begin{tabular}{lccccc}
\hline \hline $\begin{array}{l}\text { Anode } \\
\text { interlayer }\end{array}$ & $\begin{array}{c}\text { Pixel } \\
\text { area }\left(\mathrm{cm}^{2}\right)\end{array}$ & $\begin{array}{c}J_{\mathrm{SC}} \\
\left(\mathrm{mA} \mathrm{cm}^{-2}\right)\end{array}$ & $\begin{array}{c}V_{\mathrm{OC}} \\
(\mathrm{V})\end{array}$ & $\begin{array}{c}\mathrm{FF} \\
\text { PCE } \\
(\%)\end{array}$ \\
\hline $\begin{array}{l}\text { Untreated } \\
\mathrm{NiO}\end{array}$ & 0.15 & 5.90 & 0.427 & 0.51 & 1.29 \\
$\mathrm{O}_{2}$ plasma-treated $\mathrm{NiO}$ & 0.15 & 5.88 & 0.885 & 0.59 & 3.11 \\
\hline \hline
\end{tabular}

fitted parameters. The dashed colored lines denote the electron and hole quasi-Fermi-levels, while the solid black lines denote the conduction and valence band mobility edges. The charge-carrier densities as a function of activelayer thickness are shown in Figs. 2(c) and 2(d). It is clear from the simulations that the quasi-Fermi-level splitting in the middle of the active-layer bulk is expected to be almost identical in both cases. This is consistent with the expectation of little or no change in the active-layer generation and ability to split the quasi-Fermi-levels. The main difference between Figs. 2(a) and 2(b) is the position and gradient of the quasi-Fermi-levels at the anode. In Figs. 2(a) and 2(c), there are many more holes at the anode than electrons. The recombination current of electrons at the anode must be compensated by a hole current into the anode to achieve zero net current. A hole (electron) current density can be expressed as $J_{p(n)}=p(n) \mu_{p(n)} d E_{F p(n)} / d x$. Because of the high hole concentration at the anode, the gradient of the hole quasi-Fermi-level necessary to drive this current is negligible, and so the hole quasi-Fermi-level at the anode looks flat. In contrast, in the case shown in Figs. 2(b) and 2(d), the work function of the untreated $\mathrm{NiO}$ pins the quasi-Fermi-level in the middle of the gap, and the electron and hole concentrations at the anode are therefore very similar. The electron concentration is higher than in Fig. 2(c), and therefore the surface-recombination current of electrons at the anode is increased. This recombination current must now be balanced by a hole current in order to ensure zero net current at open circuit. Because of the relatively low hole concentration at the anode (relative to the case with plasma-treated $\mathrm{NiO}$ ), the gradient of the quasi-Fermi-level needed to drive this hole current is now high and clearly visible. This implies that the splitting of the quasi-Fermi-levels in the middle of the device can now be substantially higher than the open-circuit voltage which is defined by $q V_{\mathrm{OC}}=E_{F n \text { (cathode) }}-E_{F p \text { (anode). }}$. It is worth noting that, with the parameter set used, there is surface recombination present at both electrodes and in both devices, and it is the dominant recombination mechanism leading to the observed open-circuit voltage. 
TABLE II. Drift-diffusion modeling parameter list.

\begin{tabular}{|c|c|c|c|}
\hline Parameter & Symbol & Value & Unit \\
\hline & \multicolumn{3}{|c|}{ Fixed parameters } \\
\hline Effective DOS & $N_{C}, N_{V}$ & $1 \times 10^{25}$ & $\mathrm{~m}^{-3}$ \\
\hline Capture coefficient of neutral tail states & $\beta_{n, p}^{0}$ & $1 \times 10^{-16}$ & $\mathrm{~m}^{3} \mathrm{~s}^{-1}$ \\
\hline Electron injection barrier & $\varphi_{n}$ & 0.1 & $\mathrm{eV}$ \\
\hline Generation rate & $G$ & $5 \times 10^{27}$ & $\mathrm{~m}^{3} \mathrm{~s}^{-1}$ \\
\hline Temperature & $T$ & 300 & $\mathrm{~K}$ \\
\hline Surface-recombination velocity & $S_{\min }$ & $1 \times 10^{3}$ & $\mathrm{~ms}^{-1}$ \\
\hline Active-layer thickness & $d$ & $8 \times 10^{-8}$ & $\mathrm{~m}$ \\
\hline $\begin{array}{l}\text { Electron and hole mobility } \\
\text { Direct recombination coefficient }\end{array}$ & $\begin{array}{c}\mu_{n}, \mu_{p} \\
\beta_{\mathrm{dr}}\end{array}$ & $\begin{array}{l}\text { Fitted parameters } \\
5.56 \times 10^{-8} \\
3.41 \times 10^{-16}\end{array}$ & $\begin{array}{l}\mathrm{m}^{2} \mathrm{~V}^{-1} \mathrm{~s}^{-1} \\
\mathrm{~m}^{3} \mathrm{~s}^{-1}\end{array}$ \\
\hline Capture coefficient of charged tail states & $\beta_{n, p}^{+,-}$ & $1.04 \times 10^{-17}$ & $\mathrm{~m}^{3} \mathrm{~s}^{-1}$ \\
\hline Conduction and valence band tail slopes & $E_{\mathrm{cb}}, E_{\mathrm{vb}}$ & 0.107 & $\mathrm{eV}$ \\
\hline Tail state DOS & $N_{C}^{\text {tail }}, N_{V}^{\text {tail }}$ & $2.11 \times 10^{24}$ & $\mathrm{~m}^{-3} \mathrm{eV}^{-1}$ \\
\hline Effective band gap & $E_{g}$ & 1.31 & $\mathrm{eV}$ \\
\hline Hole injection barrier $\mathrm{O}_{2}$ treated & $\varphi_{p}^{\mathrm{O}_{2}}$ & 145 & $\mathrm{meV}$ \\
\hline Hole injection barrier untreated & $\varphi_{p}$ & 672 & $\mathrm{meV}$ \\
\hline Charge density dependence on $V_{\mathrm{OC}}$ & $\begin{array}{c}m^{\mathrm{O}_{2}} \\
n_{0}^{\mathrm{O}_{2}}\end{array}$ & $\begin{array}{c}\text { Experimental parameters } \\
4.372 \\
7.80 \times 10^{12}\end{array}$ & $\mathrm{~cm}^{-3}$ \\
\hline \multirow[t]{2}{*}{ Charge lifetime dependence on $V_{\mathrm{OC}}$} & $\vartheta^{\mathrm{O}_{2}}$ & 1.544 & \\
\hline & $\tau_{0}^{\mathrm{O}_{2}}$ & $6.10 \times 10^{3}$ & s \\
\hline Overall reaction order & $\delta^{\mathrm{O}_{2}}$ & 3.8 & \\
\hline \multirow[t]{2}{*}{ Charge density dependence on $V_{\mathrm{OC}}$} & $m$ & 3.413 & \\
\hline & $n_{0}$ & $1.15 \times 10^{14}$ & $\mathrm{~cm}^{-3}$ \\
\hline \multirow[t]{2}{*}{ Charge lifetime dependence on $V_{\mathrm{OC}}$} & $\vartheta$ & 1.276 & \\
\hline & $\tau_{0}$ & 0.40 & s \\
\hline Overall reaction order & $\delta$ & 3.7 & \\
\hline
\end{tabular}

\section{Simulation versus experiment}

To evaluate this device simulation, we now compare with both steady-state and transient experimental data. We start by considering the ideality factor, which has been shown to be an important parameter for understanding the dominant recombination mechanism in solar cells [16]. The ideality factor is introduced into the description of semiconductor diodes to account for deviations from the ideal diode law where the recombination current $J \approx \exp (q V / k T)$. While the ideality factor is usually determined experimentally from the slope of the dark current-voltage curve, it is typically explained and interpreted by considering the dependence of the recombination rate. In order to understand the recombination mechanism, most models attempt to explain the ideality factor $n_{\mathrm{id}}$ via the dependence of recombination rate $R$ on the splitting of the quasi-Fermilevels $\Delta E_{F}$ at one position (essentially OD models)

$$
n_{\mathrm{id}}=\frac{1}{k T} \frac{d \Delta E_{F}}{d \ln (R)} .
$$

Equation (2) allows one to directly interpret the ideality factor in terms of different recombination mechanisms. For example, Shockley-Read-Hall recombination via a deep trap in the case $n=p$ would directly lead to $n_{\text {id }}=2$ using Eq. (2). However, in practice, it is usually not possible to directly measure the recombination rate or quasi-Fermilevel splitting. Instead, we have to find measurable quantities that still allow the data to be interpreted. One way to do that is to measure the open-circuit voltage as a function of light intensity. At open circuit, no current is flowing, and so all effects of the series resistance are minimized and $\Delta E_{F}=$ $q V_{\mathrm{OC}}$ is valid at least in the bulk of the material. At open circuit, the average generation rate and the average recombination rate are identical, so the light intensity $\phi$ is directly proportional to the average recombination rate. Thus,

$$
n_{\mathrm{id}}=\frac{q}{k T} \frac{d V_{\mathrm{OC}}}{d \ln (\phi)}
$$

is a useful definition of the light ideality factor. However, while the recombination rate is a quantity that can depend on the position within the device, the light intensity is proportional only to the average recombination rate. This means the ideality factor averages over various different recombination 


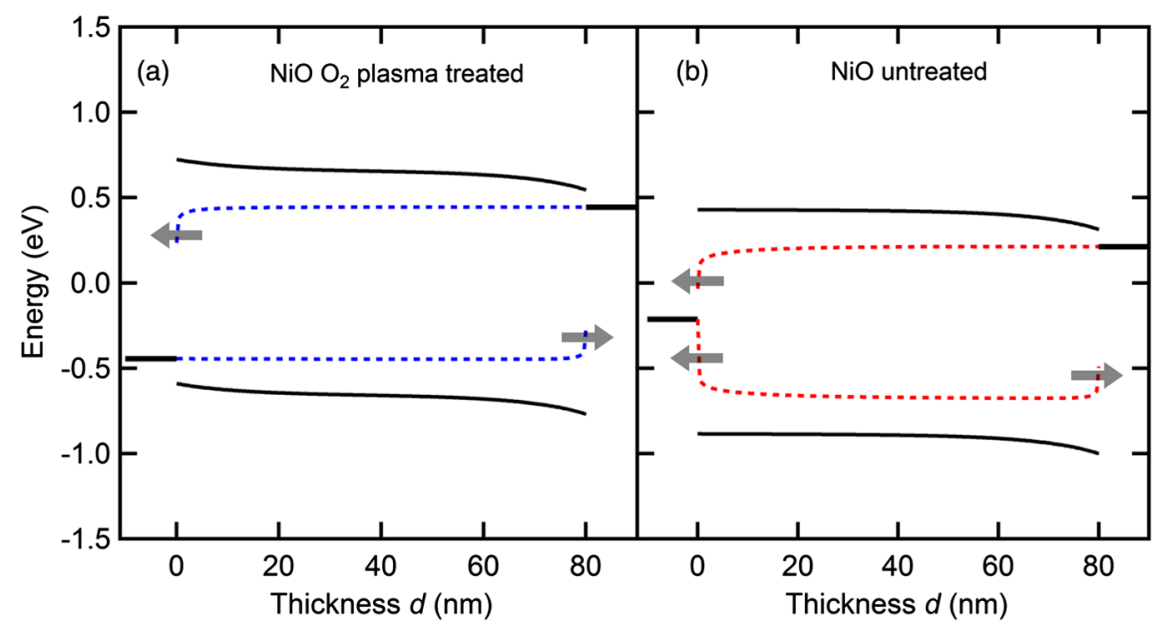

FIG. 2. Simulated band diagrams and total carrier densities as a function of active-layer thickness for $\mathrm{O}_{2}$ plasma-treated $\mathrm{NiO}$ (a),(c) and untreated $\mathrm{NiO}$ (b),(d). Band diagrams (a),(b) show the mobility edge (solid lines) and quasi-Fermi-level splitting (dashed lines), while arrows demonstrate the presence of surface recombination. (c) and (d) show electron carrier densities (filled circles) and hole carrier densities (empty circles). The anode contact is located at $0 \mathrm{~nm}$ and the cathode at $80 \mathrm{~nm}$.

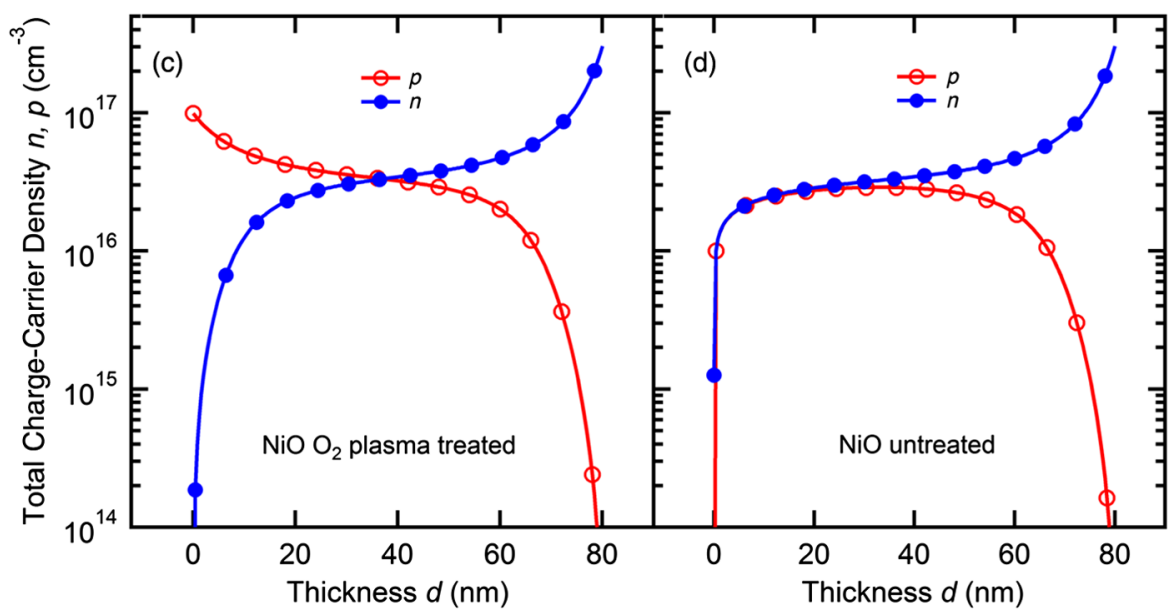

mechanisms that may dominate the ideality factor at different light intensities or open-circuit voltages.

The value of the ideality factor for OPV devices typically lies between 1 and 2, as most OPV materials exhibit a distribution of trap states extending into the electronic band gap $[16,44,45]$. A value of 1 is associated with recombination of free charges, while a value of 2 is expected for recombination of a free charge with a charge in a trap state. Surface recombination typically is expected to have an ideality factor of 1 if we assume that carriers trapped close to the electrodes cannot directly recombine at the electrode [19].

It can be seen in Fig. 3(a) that the light ideality factor is decreasing with increasing voltage for both devices; however, this occurs at a lower voltage for the untreated device, attributed to the offset between the hole transport level and the anode work function. Figure 3(b) shows the light

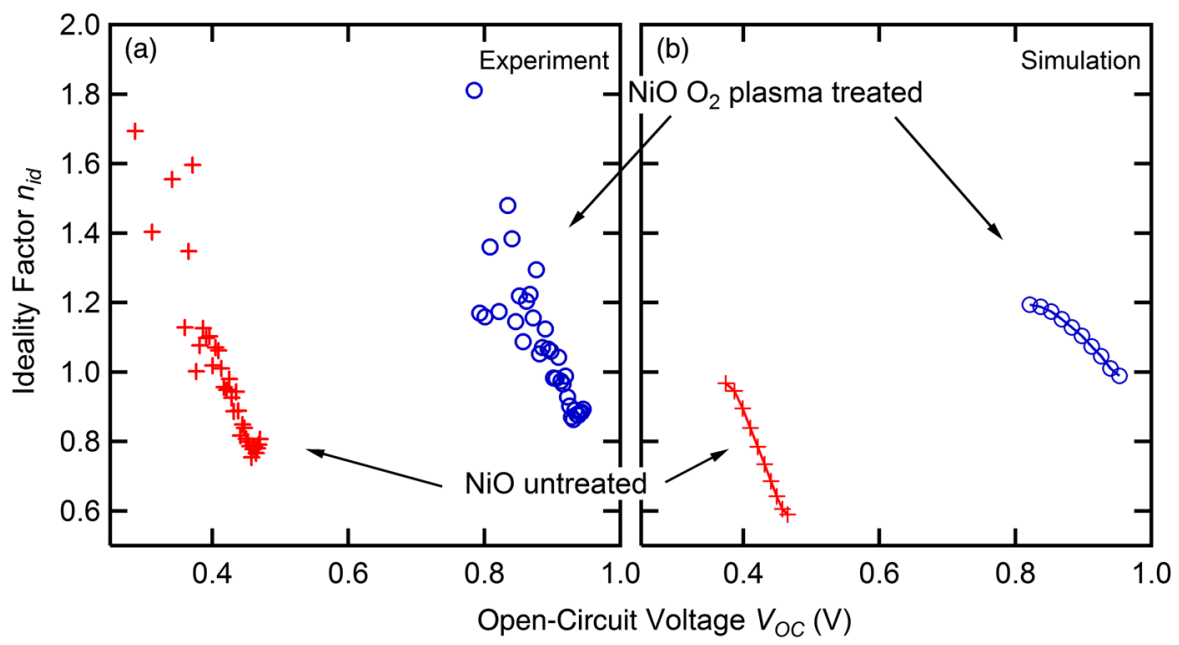

FIG. 3. Measured (a) and simulated (b) ideality factors at different open-circuit voltages for an untreated NiO-based device (crosses) and a plasmatreated NiO-based device (open circles). 
ideality factor obtained from device simulations. It appears to agree well with that measured experimentally, showing a decreasing ideality factor towards one and, in the case of the untreated device, below 1 . As only the work function of the anode is varied in the simulations, these results suggest a shift in the dominant recombination mechanism from trapassisted bulk recombination at low voltages, $n_{\text {id }}>1$, to surface free charge-carrier recombination at higher voltages, $n_{\text {id }}=1$, as observed in the band diagrams in Fig. 2.

In order to quantify the rate of recombination at open circuit, the average excess charge-carrier density is required and is measured by charge extraction. While a brief description of the experimental procedure can be found in the materials and methods section, the technique is described in detail elsewhere $[12,13,46]$. Assuming Ohmic contacts and complete collection, the charge extraction measurement measures the amount of charge required for a certain quasi-Fermi-level splitting to be achieved and is therefore used as an in situ measure of the active layer DOS. Previously $[12,13,46]$, it has been observed that the charge-carrier density measured by charge extraction in organic solar cells depends exponentially on open-circuit voltage and follows

$$
n=n_{0} \exp \left(\frac{q V_{\mathrm{OC}}}{m k T}\right)
$$

with the slope $m$ varying between different samples. Numerical simulations and experiments show that the empirical slope $m$ depends mostly on the shape of the density of trap states, on doping, and on the device thickness $[19,35,46,47]$. The experimental data at low light intensity can deviate from this behavior due to shunt resistance limitations and comparable charge density of photogenerated carriers to that of the geometric capacitance, as well as potentially poor spatial overlap of holes and electrons. For this reason, fitting parameters $m$ and $n_{0}$ are obtained at high light intensity $[13,46]$.

The experimental charge-carrier density at open circuit, measured with CE, can be seen in Fig. 4(a). The major difference between the $\mathrm{NiO}$ oxygen plasma-treated and untreated devices is a large shift in open-circuit voltage at a given charge density, corresponding to $407 \mathrm{mV}$ at a chargecarrier density of $2.0 \times 10^{16} \mathrm{~cm}^{-3}$. Such a shift could be attributed to a change in the active-layer energetics; however, a similar shift is present in the simulation results seen in Fig. 4(b). As we expect the energetic properties and
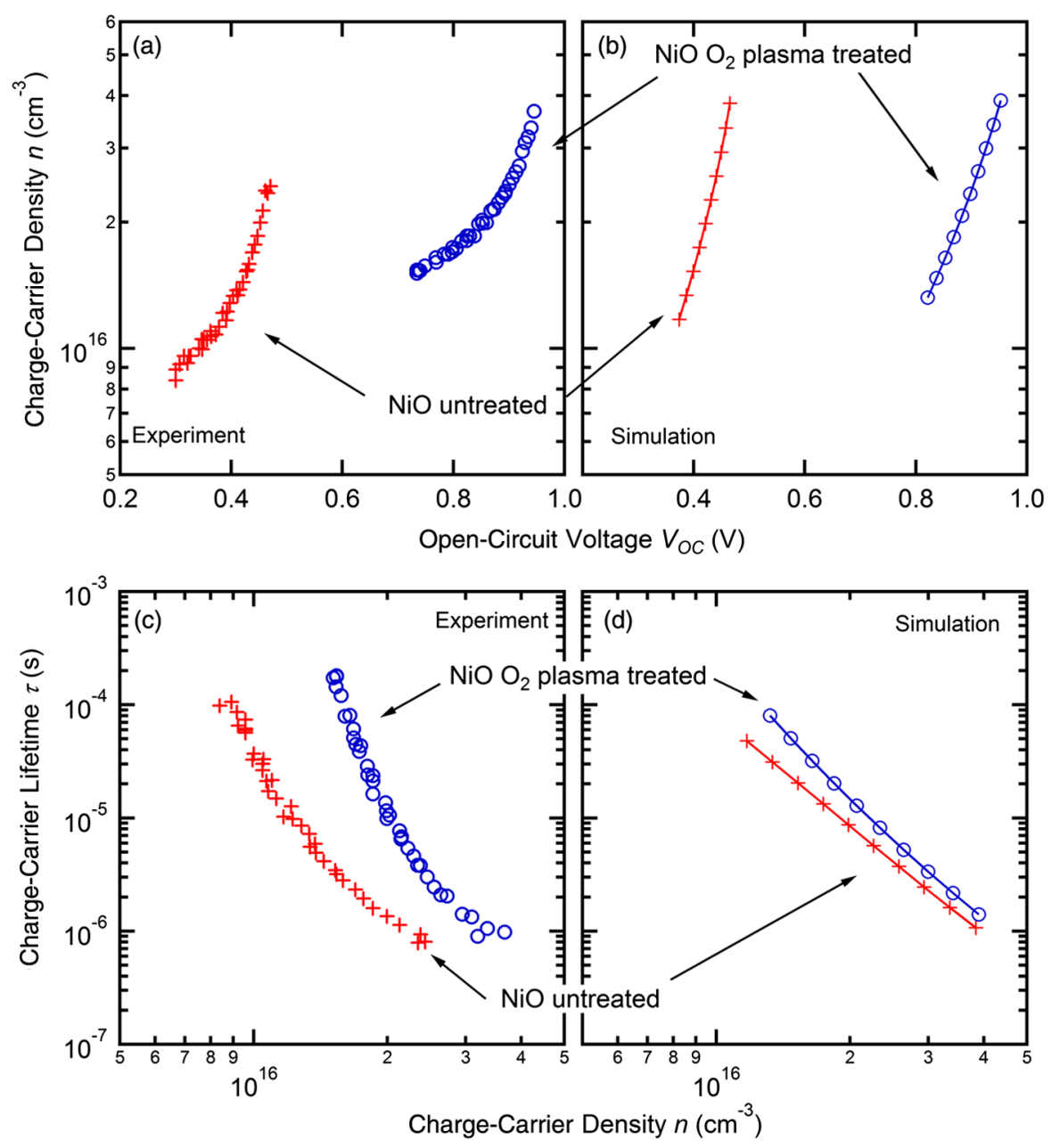

FIG. 4. Measured (a),(c) and simulated (b),(d) charge-carrier density $n$ as a function of $V_{\mathrm{OC}}$ as measured from $\mathrm{CE}$ (a),(b) and charge-carrier lifetime $\tau$ as measured from TPV (c),(d). $\mathrm{O}_{2}$ plasma-treated $\mathrm{NiO}$ (open circles) and untreated $\mathrm{NiO}$ (crosses). 
density of states of the active layer to remain the same for both devices, since the active layer is identical, we assign this shift in voltage as a result of a change in the electrode work function, as this is the only parameter varied in the simulation. The similar values for charge density in both cases is consistent with the observation that the quasiFermi-level splitting in the bulk of the devices is similar, as seen in the simulated band diagrams in Fig. 2. However, it is apparent that, in the untreated device, this bulk quasiFermi-level splitting is substantially greater than the Fermilevel splitting at the device electrodes. The open-circuit voltage measured no longer represents the splitting of the quasi-Fermi-levels in the active-layer bulk; rather, it is only a measure of the quasi-Fermi-level splitting at the electrode. Therefore, the 407-mV shift thus represents a difference in energetics between the photoactive blend adjacent to the electrode and that in the bulk of the photoactive layer.

As well as a decrease in the $V_{\mathrm{OC}}$, both the experiment and simulation show an increase in the slope $(1 / \mathrm{m})$ for the untreated device. It is possible to express the relationship between the light ideality factor $\left(n_{\mathrm{id}}\right)$ and the dimensionless parameters $(m)$ and $(\vartheta)$, which describe the voltage dependence of the carrier density [Eq. (4)] and the carrier lifetime [Eq. (5)], respectively, as $\frac{1}{n_{\mathrm{id}}}=\frac{1}{m}+\frac{1}{9}$, the derivation of which can be found in Supplemental Material [48] and elsewhere [19]. Just as surface recombination influences the ideality factor at high light intensity, we also expect it to influence the slope of $n\left(V_{\mathrm{OC}}\right)$. Therefore, in the case of poor electrode alignment, the validity of $m$ as defined in Eq. (4) as a true measure of the active-layer DOS becomes unreliable and should not be used as an assay of the activelayer DOS. For example, such a problem could arise when comparing devices fabricated during different electrode evaporations.

A change in recombination kinetics can be observed by looking at the charge density dependence of the carrier lifetime, as shown from the experiment and simulation in Figs. 4(c) and 4(d), respectively. This allows a comparison of systems with differing voltage dependence of the chargecarrier density. The total charge-carrier lifetime $\left(\tau_{n}\right)$ is given by $\delta \tau_{\Delta n}$, where $\tau_{\Delta n}$ is the lifetime of charges generated from a small laser perturbation during TPV measurements. This appears to follow the empirical relation

$$
\tau_{\Delta n}=\tau_{\Delta n_{0}} \exp \left(-\frac{q V_{\mathrm{OC}}}{\vartheta k T}\right)
$$

as reported previously $[12,13,21,35]$. The overall reaction order $\delta$ describes the $n$ dependence of nongeminate recombination and can be defined by $\delta=\frac{m}{9}+1$.

It was shown previously [12] that the $V_{\mathrm{OC}}$ could be described as the sum of an energetic component and a kinetic component such that

$$
V_{\mathrm{OC}}=\frac{1}{q}\left(I P_{D}-E A_{A}\right)-\frac{n_{\mathrm{id}} k T}{q} \ln \left(\frac{J_{\mathrm{BI}}}{J_{\mathrm{gen}}}\right),
$$

where $I P_{D}$ and $E A_{A}$ are the ionization potential and electron affinity of the fullerene and polymer, respectively (or, in this case, the energetics of the contacts), and $J_{\mathrm{BI}}$ represents the loss current at open circuit due to nongeminate recombination as measured with CE and TPV.

Figure 4(c) describes the $n$ dependence of the carrier lifetime at open circuit, as measured experimentally from TPV. There is a lengthening of the carrier lifetime in the $\mathrm{O}_{2}$ plasma-treated device, approximately 7 times longer than the untreated device at a carrier density of $2.0 \times 10^{16} \mathrm{~cm}^{-3}$. This change in recombination kinetics is expected to lead to an increase of about $\Delta V_{\mathrm{OC}} \approx \frac{n_{\mathrm{id}} k T}{q} \ln (7)=60 \mathrm{mV}$ in $V_{\mathrm{OC}}$, for an ideality factor of 1.17 . The longer carrier lifetime is in agreement with that obtained from simulations, which also suggest a lengthening of the lifetime in the $\mathrm{O}_{2}$ plasma device; however, the difference is slightly less pronounced to that seen experimentally, partly due to the simulations predicting a slightly higher charge density in the untreated device than measured experimentally.

One might expect surface recombination and bulk recombination to be linked, such that an increase in surface recombination will reduce the bulk $n$, in turn decreasing the rate of bulk recombination. It is perhaps no surprise that the voltage shift expected from the observed change in recombination is small compared to the larger shift in energetics. Combining both the $400-\mathrm{mV}$ shift from $n\left(V_{\mathrm{OC}}\right)$ and the $60-\mathrm{mV}$ shift from $\tau(n)$, our analysis is consistent with the observed change in voltage from $J-V$ measurements.

By definition, at open circuit, no external current is present; therefore, all photogenerated charges must recombine. It is possible to calculate the open-circuit voltage over the range of light intensities measured based on our of measurements of $J_{\text {loss }}$ from the charge-carrier density, lifetime, and $J_{\mathrm{SC}}$ as previously demonstrated [21]:

$$
V_{\mathrm{OC}}=\frac{m k T}{q \delta} \ln \left(\frac{\delta J_{\mathrm{SC}} \tau_{\Delta n_{0}}}{q d n_{0}}\right)
$$

Figure 1(b) shows a good correlation between the expected $V_{\mathrm{OC}}$ from measurements of charge recombination and the measured $V_{\mathrm{OC}}$ as a function of light intensity. At 1 sun equivalent, the difference between the measured $V_{\mathrm{OC}}$ and the expected $V_{\mathrm{OC}}$ is less than $5 \mathrm{mV}$. This demonstrates that the experimental techniques account for both recombination pathways, nongeminate recombination in the active layer bulk, and surface recombination at the electrodes; therefore, the successful reconstruction of the device voltage dependence with light intensity is possible. This result does, however, demonstrate the importance of understanding the effect surface recombination has on these measurements, in order that they not be misinterpreted as a change in the inherent bulk recombination of the active layer. 


\section{CONCLUSION}

In conclusion, we investigate the effects of surface recombination on measurements of recombination kinetics in OPV by combining CE and TPV experimental techniques with $1 \mathrm{D}$ drift-diffusion simulations. An $\mathrm{O}_{2}$ plasma treatment is used to vary the work function of a $\mathrm{NiO}$ hole transporting interlayer, which leads to an open-circuit voltage change of $458 \mathrm{mV}$ at 1 sun. The ideality factor is observed to decrease below 1 at high light intensity, indicating a shift in the dominant recombination mechanism from trap-assisted bulk nongeminate recombination to surface recombination at the electrodes. Measurements of charge-carrier recombination at open circuit through $\mathrm{CE}$ and TPV are successful in recreating a device open-circuit performance in the presence of surface recombination over the light intensity range studied. A reduction in the carrier lifetime for a given charge density is observed, but its effect on the open-circuit voltage is small compared to the energetic shift caused by a reduction of the quasi-Fermilevel splitting at the electrode due to recombination being localized near the electrode where the voltage is measured, rather than in the bulk. Drift-diffusion simulations are in good agreement with the experiment throughout and show the splitting of the quasi-Fermi-levels in the bulk remains similar for both devices but is reduced at the electrode due to surface recombination.

\section{MATERIALS AND METHODS}

\section{A. Device fabrication}

Devices are made with the layer configuration ITO/NiO/PCDTBT: $\mathrm{PC}_{71} \mathrm{BM} / \mathrm{Ca} / \mathrm{Al}$. Initially, the commercially available $25 \times 25 \mathrm{~mm}^{2}$ ITO-coated glass substrates are subjected to a standard solvent cleaning sequence of detergent, water, acetone, and propan-2-ol followed by ultraviolet ozone plasma treatment. The $\mathrm{NiO}$ hole transporting layer is spin coated (spin rate, $4000 \mathrm{rpm}$; acceleration, $10000 \mathrm{rpm} / \mathrm{s}$; time, $40 \mathrm{~s}$ ) from a nickel acetate tetrahydrate precursor $(0.2 \mathrm{M}$ in $1: 0.012$ ratio 2 -methoxyethanol:ethanolamine solvent mixture) and annealed in air at $250^{\circ} \mathrm{C}$ for $30 \mathrm{~min}$. After annealing, $\mathrm{NiO}$ layers are exposed to oxygen plasma treatment at $155 \mathrm{~W}$ at 0.08 Torr for periods between 0 and $8 \mathrm{~min}$. This is followed by deposition of a poly[N-900-hepta-decanyl-2,7-carbazole-alt-5,5-(40,70di-2-thienyl-20,10,30-benzothiadiazole)] (PCDTBT) and $[6,6]$-phenyl C71-butyric acid methyl ester $\left(\mathrm{PC}_{71} \mathrm{BM}\right)$ $\left(1: 2\right.$ blend ratio, $\left.30 \mathrm{mg} \mathrm{ml}^{-1}\right)$ active layer by spin coating from a chlorobenzene solution (spin rate, $4000 \mathrm{rpm}$; acceleration, $2000 \mathrm{rpm} / \mathrm{s}$; time, $10 \mathrm{~s}$, giving a thickness of $80 \mathrm{~nm}$ ). Fabrication is completed by evaporation of a 100-nm-thick $\mathrm{Al}$ electrode, preceded by $20 \mathrm{~nm}$ of Ca.

\section{B. CE and TPV}

Charge extraction, transient photocurrent, and transient photovoltage measurements and analysis are carried out as previously reported [13]. For CE and TPV measurements, white-light illumination is provided by a ring of 12 white LEDs capable of a power up to 7 suns, calibrated to device performance as measured under simulated AM1.5 illumination. During CE, the device is held at open circuit under varied illumination intensities for $500 \mathrm{~ms}$ to allow the system to reach a steady state. Simultaneously, the light source is switched off (approximately $200 \mathrm{~ns}$ ) and the device switched to short circuit. The resulting current transient is measured across a $50-\Omega$ resistor on a Tektronix TDS3032 oscilloscope and integrated to obtain the excess charge-carrier density following a correction for the capacitive charge on the electrode and recombination losses during extraction. During TPV and transient photocurrent (TPC), background illumination equivalent to that used in CE is provided by the LEDs, while the pulsed excitation is provided with a nitrogen-dye-pumped laser with a fixed wavelength pump source. The excitation wavelength used is $610 \mathrm{~nm}$. Voltage transients are measured on a Tektronix TDS3032 oscilloscope and fitted with a monoexponential to obtain the small perturbation carrier lifetime.

\section{Device modeling}

1D drift-diffusion simulations are performed as previously described $[19,36,38,49]$, by solving three coupled differential equations: the Poisson equation and continuity equations for electrons and holes. Bulk recombination is treated as a sum of direct, as calculated from Langevin theory [50], and trap-assisted recombination, calculated from the tail recombination model as described previously [36]. The boundary conditions for current density at the contact is given by $J_{\text {contact }(n, p)}=q S\left(n, p-n_{0}, p_{0}\right)$, where $S$ is the surface-recombination velocity, $n_{0}$ and $p_{0}$ are the equilibrium concentrations related to the injection barriers, and $n$ and $p$ are carrier concentrations related to the quasiFermi-level splitting. More detailed equations can be found in Supplemental Material [51]. To compare with the CE experiment, the charge density is calculated as follows, where $x$ is the spatial coordinate, $d$ is the active layer thickness, and $\xi_{b} b$ is the blackbody photon flux:

$$
\begin{aligned}
n_{\mathrm{CE}}= & \frac{1}{d} \int_{0}^{d} n_{\mathrm{AL}}(V, \xi, x)-n_{\mathrm{SC}, \mathrm{dark}}\left(0, \xi_{b b}, x\right) d x \\
& +n_{\text {electrode }}-n_{\text {capcor }} .
\end{aligned}
$$

The first term is the total charge in the active layer, the second term subtracts the charge present at short circuit in the dark, and the third term corrects for the effects of charge density on the electrodes, while the last term subtracts the geometric capacitance as is done experimentally. The charge lifetime at open circuit, to compare with TPV, is given by the ratio of the excess carrier density and recombination rate $(R) \tau_{n}=\frac{n_{\mathrm{CE}}}{R}$. A downhill simplex fitting routine is used to minimize the difference between 
the experimental and simulated results for the $\mathrm{O}_{2}$ plasmatreated device, after which only the position of the electrode work function is changed.

\section{ACKNOWLEDGMENTS}

J. R. D. and S. W. acknowledge funding from Engineering and Physical Sciences Research Council (EPSRC) Projects No. EP/J500021, No. EP/I019278, and No. EP/G037515 via the Doctoral Training Centre in Science and Application of Plastic Electronic Materials at Imperial College London. T. K. acknowledges support by an Imperial College Junior Research Fellowship and support from the Deutsche Forschungsgemeinschaft (DFG) (Grant No. KI-1571/2-1) and from the Helmholtz Association via the Helmholtz Energy Alliance Hybrid PV. J. N. acknowledges the Royal Society for a Wolfson Merit Award and the Engineering and Physical Sciences Research Council for support via Grants No. EP/K029843/1, No. EP/G031088/1, and No. EP/ J017361/1. The authors also thank Sachetan Tuladhar for help with Kelvin probe measurements.

[1] G. Dennler, M. C. Scharber, and C. J. Brabec, Polymerfullerene bulk-heterojunction solar cells, Adv. Mater. 21, 1323 (2009).

[2] G. Li, R. Zhu, and Y. Yang, Polymer solar cells, Nat. Photonics 6, 153 (2012).

[3] Y. Liu, J. Zhao, Z. Li, C. Mu, W. Ma, H. Hu, K. Jiang, H. Lin, H. Ade, and H. Yan, Aggregation and morphology control enables multiple cases of high-efficiency polymer solar cells, Nat. Commun. 5, 5293 (2014).

[4] Z. He, C. Zhong, S. Su, M. Xu, H. Wu, and Y. Cao, Enhanced power-conversion efficiency in polymer solar cells using an inverted device structure, Nat. Photonics 6, 591 (2012).

[5] M. A. Green, K. Emery, Y. Hishikawa, W. Warta, and E. D. Dunlop, Solar cell efficiency tables (version 43), Prog. Photovoltaics 22, 1 (2014).

[6] X. Guo, N. Zhou, S. J. Lou, J. Smith, D. B. Tice, J. W. Hennek, R. P. Ortiz, J. T. L. Navarrete, S. Li, J. Strzalka, L. X. Chen, R. P. H. Chang, A. Facchetti, and T. J. Marks, Polymer solar cells with enhanced fill factors, Nat. Photonics 7, 825 (2013).

[7] E. L. Ratcliff, B. Zacher, and N. R. Armstrong, Selective interlayers and contacts in organic photovoltaic cells, J. Phys. Chem. Lett. 2, 1337 (2011).

[8] K. X. Steirer, P. F. Ndione, N. E. Widjonarko, M. T. Lloyd, J. Meyer, E. L. Ratcliff, A. Kahn, N. R. Armstrong, C. J. Curtis, D. S. Ginley, J. J. Berry, and D. C. Olson, Enhanced efficiency in plastic solar cells via energy matched solution processed niox interlayers, Adv. Energy Mater. 1, 813 (2011).

[9] M. C. Scharber, D. Mühlbacher, M. Koppe, P. Denk, C. Waldauf, A. J. Heeger, and C. J. Brabec, Design rules for donors in bulk-heterojunction solar cells-Towards $10 \%$ energy-conversion efficiency, Adv. Mater. 18, 789 (2006).
[10] K. Vandewal, A. Gadisa, W. D. Oosterbaan, S. Bertho, F. Banishoeib, I. Van Severen, L. Lutsen, T. J. Cleij, D. Vanderzande, and J.V. Manca, The relation between open-circuit voltage and the onset of photocurrent generation by charge-transfer absorption in polymer:fullerene bulk heterojunction solar cells, Adv. Funct. Mater. 18, 2064 (2008).

[11] V. D. Mihailetchi, L. J. A. Koster, and P. W. M. Blom, Effect of metal electrodes on the performance of polymer:fullerene bulk heterojunction solar cells, Appl. Phys. Lett. 85, 970 (2004).

[12] D. Credgington and J. R. Durrant, Insights from transient optoelectronic analyses on the open-circuit voltage of organic solar cells, J. Phys. Chem. Lett. 3, 1465 (2012).

[13] D. Credgington, R. Hamilton, P. Atienzar, J. Nelson, and J. R. Durrant, Non-geminate recombination as the primary determinant of open-circuit voltage in polythiophene: fullerene blend solar cells: an analysis of the influence of device processing conditions, Adv. Funct. Mater. 21, 2744 (2011).

[14] D. Rauh, A. Wagenpfahl, C. Deibel, and V. Dyakonov, Relation of open circuit voltage to charge carrier density in organic bulk heterojunction solar cells, Appl. Phys. Lett. 98, 133301 (2011).

[15] R. Xia, D.-S. Leem, T. Kirchartz, S. Spencer, C. Murphy, Z. He, H. Wu, S. Su, Y. Cao, J. S. Kim, J. C. deMello, D. D. C. Bradley, and J. Nelson, Investigation of a conjugated polyelectrolyte interlayer for inverted polymer: fullerene solar cells, Adv. Energy Mater. 3, 718 (2013).

[16] T. Kirchartz, F. Deledalle, P. S. Tuladhar, J. R. Durrant, and J. Nelson, On the differences between dark and light ideality factor in polymer:fullerene solar cells, J. Phys. Chem. Lett. 4, 2371 (2013).

[17] J. Reinhardt, M. Grein, C. Bühler, M. Schubert, and U. Würfel, Identifying the impact of surface recombination at electrodes in organic solar cells by means of electroluminescence and modeling, Adv. Energy Mater. 4, 1400081 (2014).

[18] K. M. Knesting, H. Ju, C. W. Schlenker, A. J. Giordano, A. Garcia, O. L. Smith, D. C. Olson, S. R. Marder, and D. S. Ginger, ITO interface modifiers can improve $V_{\mathrm{OC}}$ in polymer solar cells and suppress surface recombination, J. Phys. Chem. Lett. 4, 4038 (2013).

[19] T. Kirchartz and J. Nelson, Meaning of reaction orders in polymer:fullerene solar cells, Phys. Rev. B 86, 165201 (2012).

[20] A. Guerrero, L. F. Marchesi, P. P. Boix, S. Ruiz-Raga, T. Ripolles-Sanchis, G. Garcia-Belmonte, and J. Bisquert, How the charge-neutrality level of interface states controls energy level alignment in cathode contacts of organic bulk-heterojunction solar cells, ACS Nano 6, 3453 (2012).

[21] S. A. Hawks, F. Deledalle, J. Yao, D. G. Rebois, G. Li, J. Nelson, Y. Yang, T. Kirchartz, and J. R. Durrant, Relating recombination, density of states, and device performance in an efficient polymer:fullerene organic solar cell blend, Adv. Energy Mater. 3, 1201 (2013).

[22] A. Foertig, J. Kniepert, M. Gluecker, T. Brenner, V. Dyakonov, D. Neher, and C. Deibel, Nongeminate and 
geminate recombination in PTB7:PCBM solar cells, Adv. Funct. Mater. 24, 1306 (2014).

[23] D. Rauh, C. Deibel, and V. Dyakonov, Charge density dependent nongeminate recombination in organic bulk heterojunction solar cells, Adv. Funct. Mater. 22, 3371 (2012).

[24] S. Albrecht, S. Janietz, W. Schindler, J. Frisch, J. Kurpiers, J. Kniepert, S. Inal, P. Pingel, K. Fostiropoulos, N. Koch, and D. Neher, Fluorinated copolymer pcpdtbt with enhanced open-circuit voltage and reduced recombination for highly efficient polymer solar cells, J. Am. Chem. Soc. 134, 14932 (2012).

[25] J. C. Blakesley and D. Neher, Relationship between energetic disorder and open-circuit voltage in bulk heterojunction organic solar cells, Phys. Rev. B 84, 075210 (2011).

[26] I. Lange, J. Kniepert, P. Pingel, I. Dumsch, S. Allard, S. Janietz, U. Scherf, and D. Neher, Correlation between the open circuit voltage and the energetics of organic bulk heterojunction solar cells, J. Phys. Chem. Lett. 4, 3865 (2013).

[27] J. W. Ryan, T. Kirchartz, A. Viterisi, J. Nelson, and E. Palomares, Understanding the effect of donor layer thickness and $\mathrm{a}_{3} \mathrm{O}_{3}$ hole transport layer on the open-circuit voltage in Squaraine/C60 bilayer solar cells, J. Phys. Chem. C 117, 19866 (2013).

[28] S. R. Cowan, J. V. Li, D. C. Olson, and E. L. Ratcliff, Contact-induced mechanisms in organic photovoltaics: A steady-state and transient study, Adv. Energy Mater. 5, 1400549 (2015).

[29] T. Kirchartz, B. E. Pieters, K. Taretto, and U. Rau, Mobility dependent efficiencies of organic bulk heterojunction solar cells: Surface recombination and charge transfer state distribution, Phys. Rev. B 80, 035334 (2009).

[30] A. Wagenpfahl, C. Deibel, and V. Dyakonov, Organic solar cell efficiencies under the aspect of reduced surface recombination velocities, IEEE J. Sel. Top. Quantum Electron. 16, 1759 (2010).

[31] A. Wagenpfahl, D. Rauh, M. Binder, C. Deibel, and V. Dyakonov, S-shaped current-voltage characteristics of organic solar devices, Phys. Rev. B 82, 115306 (2010).

[32] K. Kawano, R. Pacios, D. Poplavskyy, J. Nelson, D. D. C. Bradley, and J. R. Durrant, Degradation of organic solar cells due to air exposure, Sol. Energy Mater. Sol. Cells 90, 3520 (2006).

[33] M. Jørgensen, K. Norrman, and F. C. Krebs, Stability/ degradation of polymer solar cells, Sol. Energy Mater. Sol. Cells 92, 686 (2008).

[34] M. D. Irwin, D. B. Buchholz, A. W. Hains, R. P. H. Chang, and T.J. Marks, p-type semiconducting nickel oxide as an efficiency-enhancing anode interfacial layer in polymer bulk-heterojunction solar cells, Proc. Natl. Acad. Sci. U.S.A. 105, 2783 (2008).

[35] A. Foertig, J. Rauh, V. Dyakonov, and C. Deibel, Shockley equation parameters of P3HT:PCBM solar cells determined by transient techniques, Phys. Rev. B 86, 115302 (2012).

[36] T. Kirchartz, B. E. Pieters, J. Kirkpatrick, U. Rau, and J. Nelson, Recombination via tail states in polythiophene: fullerene solar cells, Phys. Rev. B 83, 115209 (2011).
[37] C. G. Shuttle, B. O’Regan, A. M. Ballantyne, J. Nelson, D. D. C. Bradley, J. de Mello, and J.R. Durrant, Experimental determination of the rate law for charge carrier decay in a polythiophene: Fullerene solar cell, Appl. Phys. Lett. 92, 093311 (2008).

[38] S. Schäfer, A. Petersen, T. A. Wagner, R. Kniprath, D. Lingenfelser, A. Zen, T. Kirchartz, B. Zimmermann, U. Würfel, X. Feng, and T. Mayer, Influence of the indium tin oxide/organic interface on open-circuit voltage, recombination, and cell degradation in organic small-molecule solar cells, Phys. Rev. B 83, 165311 (2011).

[39] See Supplemental Material at http://link.aps.org/ supplemental/10.1103/PhysRevApplied.4.024020 for a discussion of $\mathrm{S}$-shaped $J-V$ curves as a consequence of a reduced minority surface-recombination velocity.

[40] K. X. Steirer, J. P. Chesin, N. E. Widjonarko, J. J. Berry, A. Miedaner, D. S. Ginley, and D. C. Olson, Solution deposited nio thin-films as hole transport layers in organic photovoltaics, Org. Electron. 11, 1414 (2010).

[41] See Supplemental Material at http://link.aps.org/ supplemental/10.1103/PhysRevApplied.4.024020 for Kelvin probe data.

[42] See Supplemental Material at http://link.aps.org/ supplemental/10.1103/PhysRevApplied.4.024020 for a comparison of the linearity of $J_{\mathrm{SC}}$ with light intensity.

[43] C. Law, L. Miseikis, S. Dimitrov, P. Shakya-Tuladhar, X. Li, P. R. F. Barnes, J. Durrant, and B. C. O'Regan, Performance and stability of lead perovskite/ $\mathrm{TiO}_{2}$, polymer/PCBM, and dye sensitized solar cells at light intensities up to 70 suns, Adv. Mater. 26, 6268 (2014).

[44] G.-J. A. H. Wetzelaer, M. Kuik, and P. W. M. Blom, Identifying the nature of charge recombination in organic solar cells from charge-transfer state electroluminescence, Adv. Energy Mater. 2, 1232 (2012).

[45] R. A. Street, M. Schoendorf, A. Roy, and J. H. Lee, Interface state recombination in organic solar cells, Phys. Rev. B 81, 205307 (2010).

[46] F. Deledalle, P. S. Tuladhar, J. Nelson, J. R. Durrant, and T. Kirchartz, Understanding the apparent charge density dependence of mobility and lifetime in organic bulk heterojunction solar cells, J. Phys. Chem. C 118, 8837 (2014).

[47] F. Deledalle, T. Kirchartz, M. S. Vezie, M. CampoyQuiles, P. S. Tuladhar, J. Nelson, and J. R. Durrant, Understanding the Effect of Unintentional Doping on Transport Optimization and Analysis in Efficient Organic Bulk-Heterojunction Solar Cells, Phys. Rev. X 5, 011032 (2015).

[48] See Supplemental Material at http://link.aps.org/ supplemental/10.1103/PhysRevApplied.4.024020 for a derivation of the relationship of $n_{\text {id }}$ to $(m)$ and $(\vartheta)$.

[49] A. Petersen, T. Kirchartz, and T. A. Wagner, Charge extraction and photocurrent in organic bulk heterojunction solar cells, Phys. Rev. B 85, 045208 (2012).

[50] P. Langevin, Recombinaison et mobilites des ions dans les gaz, Ann. Chim. Phys. 28, 433 (1903).

[51] See Supplemental Material at http://link.aps.org/ supplemental/10.1103/PhysRevApplied.4.024020 for detailed equations used for the drift-diffusion simulations. 